\title{
Cell growth inhibitory effects of polyphenols with naphthalene skeleton against cisplatin-resistant ovarian cancer cells
}

\author{
Soon Young Shin ${ }^{1} \cdot$ Youngshim Lee ${ }^{2} \cdot$ Jihyun Park $^{2}$ • \\ Doseok Hwang ${ }^{2}$ Geunhyeong $\mathrm{Jo}^{2} \cdot \mathrm{Ji}$ Hye Lee ${ }^{3}$. \\ Dongsoo $\mathrm{Koh}^{3} \cdot$ Yoongho $\mathrm{Lim}^{2}$
}

Received: 31 August 2018/Accepted: 20 September 2018/Published online: 5 October 2018

(C) The Korean Society for Applied Biological Chemistry 2018

\begin{abstract}
Cisplatin often shows the drug resistance which could limit the chemotherapeutic efficacy. Thus, it is necessary to develop anticancer agents against cisplatin-resistant cancer cells. To identify pharmacophores exhibiting the cell growth inhibitory effect against cisplatin-resistant A2780/Cis ovarian cancer cells, we prepared 35 synthetic polyphenols bearing naphthalene skeleton including naphthalenyl chalcones, naphthalenyl flavones, naphthalenyl flavanones, 4,5-dihydro-1 $H$-pyrazol-3-yl)naphthalen-2-ols, naphthalen-1-yl- $N$-phenyl-4,5-dihydro- $1 H$-pyrazole-1-carbothioamides, and 4,5-dihydro- $1 H$-pyrazol-3-yl)naphthalen-1-ol. The correlation between their inhibitory effects and structural properties was evaluated using hologram quantitative structure activity relationship and comparative molecular field analysis. The pharmacophores derived here can lead us to design new polyphenols against the growth of cisplatin-resistant cells.
\end{abstract}

Keywords Cisplatin-resistant ovarian cancer . Clonogenicity - Comparative molecular field analysis . Hologram quantitative structure activity relationship . Polyphenols

Electronic supplementary material The online version of this article (https://doi.org/10.1007/s13765-018-0403-3) contains supplementary material, which is available to authorized users.

Yoongho Lim

yoongho@konkuk.ac.kr

1 Department of Biological Sciences, Konkuk University, Seoul 05029, Republic of Korea

2 Division of Bioscience and Biotechnology, BMIC, Konkuk University, Seoul 05029, Republic of Korea

3 Department of Applied Chemistry, Dongduk Women's University, Seoul 02748, Republic of Korea

\section{Introduction}

Cisplatin has been used for the chemotherapy of various cancers including ovarian cancer $[1,2]$. However, it often shows the drug resistance, which could limit the chemotherapeutic efficacy [3]. The patients with neoplastic lesions showed a resistance against the cytotoxicity of cisplatin [4]. Even though its complete mechanism has not been elucidated, the imperfection of DNA repair pathways raises a doubt for its reason [5]. Thus, it is necessary to develop anticancer agents against cisplatin-resistant cancer cells. Previously, we have demonstrated that polyphenols containing a cinnamaldehyde structure displayed anti-tumor activity in cisplatin-resistant ovarian cancer cells [6]. However, the inhibitory effect of naphthylated polyphenols on the growth of cisplatin-resistant cells remains unclear. Here, we prepared 35 synthetic polyphenols bearing naphthalene skeleton including naphthalenyl chalcones, naphthalenyl flavones, naphthalenyl flavanones, 4,5-dihydro-1 $H$-pyrazol-3-yl)naphthalen-2-ols, naphthalen-1-yl- $N$ phenyl-4,5-dihydro-1 $H$-pyrazole-1-carbothioamides, and 4,5-dihydro- $1 H$-pyrazol-3-yl)naphthalen-1-ol (Table S1). To identify pharmacophores exhibiting the cancer cell growth inhibitory effect (GI) against cisplatin-resistant A2780/Cis ovarian cancer cells, the correlation between structural properties of 35 synthetic polyphenols and their GI values was evaluated using hologram quantitative structure activity relationship (HQSAR) and comparative molecular field analysis (CoMFA). The pharmacophores derived here can lead us to design new polyphenols against the growth of cisplatin-resistant cells. 


\section{Materials and methods}

The experiments to obtain, culture, and maintain the human cisplatin-resistant A2780/Cis ovarian cancer cells followed the methods reported previously [6]. Thirty-five synthetic polyphenols bearing naphthalene skeleton were prepared by the methods reported previously [7-10]. The GI values against cisplatin-resistant A2780/Cis ovarian cancer cells were measured using the clonogenic assay at four different concentrations $(0,5,10$, and $20 \mu \mathrm{M})$ based on the methods reported previously [11]. The half-maximal cell growth inhibitory concentration $\left(\mathrm{GI}_{50}\right)$ values were obtained as described previously [12]. All experiments were carried out by three times iteration. HQSAR was carried out using the QSAR/HQSAR module (Sybyl software, Tripos, St. Louis, MO) on Linux PC. Since HQSAR can be calculated using the molecular fragments, the two-dimensional structures of 35 polyphenols bearing naphthalene skeleton were obtained using the Sybyl/Sketch module (Tripos). Among 35 compounds used here, seven compounds $(\mathbf{2}, \mathbf{5}, \mathbf{6}, \mathbf{1 4}, \mathbf{1 5}$, 26, and 30) were used as the test set to validate the HQSAR model. The test set selected arbitrarily by hierarchical clustering analysis (Figure S1) includes (E)-1-(1-hydroxynaphthalen-2-yl)-3-(3-methoxyphenyl) prop-2-en-1-one (derivative 2), (E)-1-(1-hydroxynaphthalen-2-yl)-3-phenylprop-2-en-1-one (derivative 5), (E) - 1 - (1-hy drox y naphthalen-2-yl)-3-(2,4,5-trimethoxyphenyl)prop-2-en-1-one (derivative 6), 3-(2,3-dimethoxyphenyl)-2,3-dihydro- $1 H$-benzo[f]chromen-1-one (derivative 14), 3-(2,4-dimethoxyphenyl)-2,3-dihydro- $1 H$ benzo[f]chromen-1-one (derivative 15), 1-(5-(2,4-dimethoxyphenyl)-1-(4-fluorophenyl)-4,5-dihydro- $1 H$-pyrazol-3yl)naphthalen-2-ol (derivative 26), and 3-(2-hydroxynaphthalen-1-yl)-5-(2-methoxyphenyl)- $N$-phenyl-4,5-dihydro-1H-pyrazole-1-carbothioamide (derivative 30). CoMFA was performed using the QSAR/CoMFA module (Tripos). Twenty-eight compounds except seven compounds chosen for the test set were used as the training set to generate the CoMFA model. The three-dimensional (3D) structures of the polyphenols for the CoMFA calculations were determined based on the 3D structures reported in PubChem (https://pubchem.ncbi.nlm.nih.gov) and their structural modifications followed the methods published previously [13]. The detailed experiments for HQSAR and CoMFA followed the methods published previously [14].

\section{Results and discussion}

The cancer cell growth inhibitory effects of 35 polyphenols bearing naphthalene skeleton measured using the clonogenicity are shown in Fig. 1, and their $\mathrm{GI}_{50}$ values are listed in Table S1. The derivative showing the best $\mathrm{GI}_{50}$ value $(2.60 \mu \mathrm{M})$ was derivative 35,5 -(2,3-dimethoxyphenyl)-3-(1-hydroxynaphthalen-2-yl)- $N$-(3,4,5-trimethoxyphenyl)-4,5-dihydro- $1 H$-pyrazole-1-carbothioamide. All derivatives contained in the training set were aligned well (Figure S2). In order to obtain the correlation between structural properties of 35 synthetic polyphenols and their $\mathrm{GI}_{50}$ values against cisplatin-resistant $\mathrm{A} 2780 / \mathrm{Cis}$ ovarian cancer cells, the partial least squares regression method was used. For the refinement, the region focusing method was used. Of several CoMFA models, a model showing the best cross-validation correlation coefficient (0.746) was selected. Its statistical data are listed in Table S2. This model gave the steric and electrostatic field values of 0.587 and 0.413 , respectively. Using this model, the $\mathrm{GI}_{50}$ values of 28 derivatives in the training set were predicted (Table S3) and compared with experimental $\mathrm{GI}_{50}$ values (Figure S3). The residuals between two values ranged from 0.17 to 10.29 . To confirm the reliability of the model, the $\mathrm{GI}_{50}$ values of seven derivatives in the test set were predicted (Table S3). The residuals between the experimental $\mathrm{GI}_{50}$ values and the predicted values ranged from 2.11 to 21.84. Therefore, the CoMFA model obtained here was concluded to be reliable. Its contour maps to visualize the steric and electrostatic field descriptors were generated as described previously (Figure S4), where derivative $\mathbf{3 5}$ was used as a template molecule [15]. These contour maps give the reason why the direction of naphthalene skeleton is important for the activity. The derivatives in the first group (1-6) have the same direction of naphthalene skeleton as that of derivative $\mathbf{3 5}$ and showed middle range of $\mathrm{GI}_{50}$ values. On the other hand, derivatives 7 and 11-21 having 2-hydroxynaphthalene-1-yl structure and showed poor activities. Derivatives 24-29 have the same naphthalene structure but showed middle range of $\mathrm{GI}_{50}$ values from 13.58 to $17.49 \mu \mathrm{M}$. This is because a bulky group at $\mathrm{N}-1$ position such as chlorophenyl or fluorophenyl would generate positive effects although the 2-hydroxynaphthalene-1-yl causes negative effects to the activity. In the next group, four derivatives (30-33) had 2-hydroxynaphthalene-1-yl but showed better activities than previous group of derivatives (24-29) generally. In this group of derivatives, bulkier group with additional carbothioamide at $N$-1 induces the activities. Both derivatives $\mathbf{3 4}$ and $\mathbf{3 5}$ have 1-hydroxynaphthalene-2-yl, but their $\mathrm{GI}_{50}$ values are different. Derivative $\mathbf{3 5}$ had much higher activity compared to the activity of derivative 34 . The only difference between two derivatives is $N$-(3,4,5-trimethoxyphenyl)-carbothioamide moiety at $N-1$. The derivatives which contain carbothioamide group (30-33, and 35) showed better $\mathrm{GI}_{50}$ values compared to the $\mathrm{GI}_{50}$ values of derivatives (24-29) which do not have the group. Therefore, the carbothioamide group and the direction of naphthalene skeleton would have an important role in the cancer 

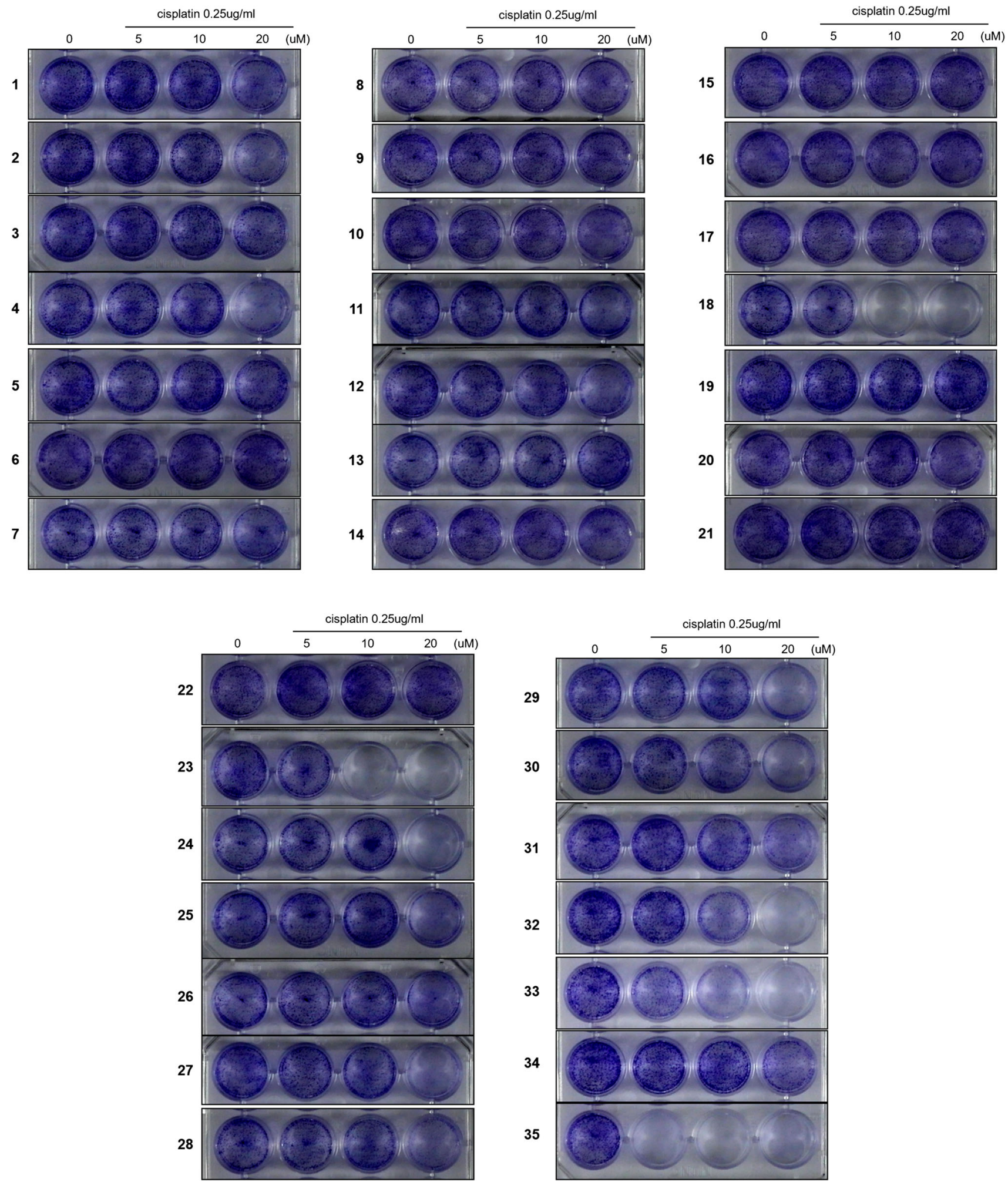

Fig. 1 The cell growth inhibitory effects of 35 polyphenols bearing naphthalene skeleton against cisplatin-resistant A2780/Cis ovarian cancer cells using the clonogenic long-term survival assay at four different concentrations, $0,5,10$, and $20 \mu \mathrm{M}$

cell growth inhibitory effects. Likewise, the electrostatic field descriptors could be separated into the electropositive group favored region (16\%) and the electronegative group favored region (84\%) (Figure S4). For the derivatives 1-23, the electrostatic effects were not significant. Derivatives 25-35 have methoxy group at ortho position in the phenyl 
Fig. 2 Structural conditions to show better cell growth inhibitory effects against cisplatin-resistant A2780/Cis ovarian cancer cells

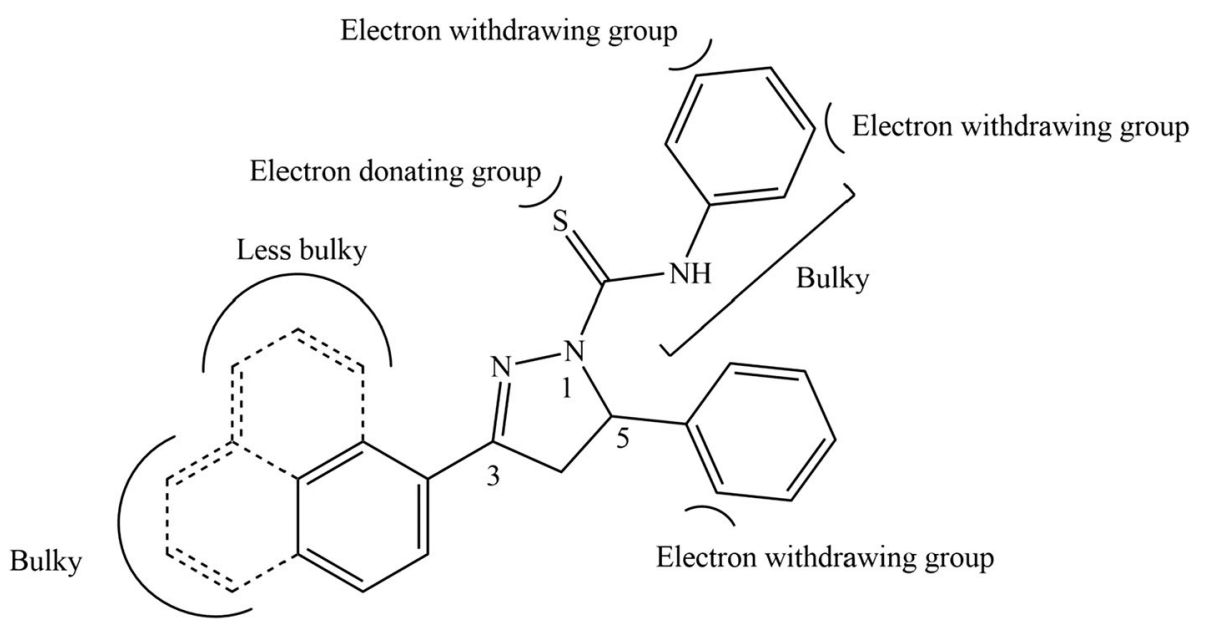

ring substituted at C-5 of pyrazole and generally showed good cytotoxic activities. Also, the methoxy groups at meta-positions of the other phenyl ring connected to the carbothioamide were colored in blue. Derivatives $\mathbf{3 3}$ and 35 which have these methoxy substituents showed the best $\mathrm{GI}_{50}$ values as $7.63 \mu \mathrm{M}$ and $2.60 \mu \mathrm{M}$, respectively. Based on electrostatic field maps, positive-charged protons of the methoxy groups seem to increase activities. Two derivatives (34 and 35) share the identical structure except substituent at $N-1$ which were $\mathrm{H}$ for derivative 34 and $N-3,4,5-$ trimethoxyphenyl carbothioamide for derivative 35. Comparison of these two derivatives supports the previous idea that negative-charged carbothioamide and positively charged methoxy protons at meta-positions affected cytotoxic activity positively.

Unlike CoMFA, because HQSAR can be built based on the fragments of the molecules, it does not require the $3 \mathrm{D}$ structures. Therefore, the sketch structures of 35 polyphenols bearing naphthalene skeleton were used for HQSAR. Like CoMFA, seven derivatives including $2,5, \mathbf{6}, \mathbf{1 4}, \mathbf{1 5}$, $\mathbf{2 6}$, and $\mathbf{3 0}$ were selected as the test set. Its statistical data are listed in Table $\mathrm{S} 4$. The $\mathrm{GI}_{50}$ values of 28 derivatives in the training set predicted using this HQSAR (Table S5) were compared with the $\mathrm{GI}_{50}$ values obtained from the in vitro experiments (Figure S5). The residuals between two values ranged from 0.53 to 24.9 . To confirm the reliability of this model, the $\mathrm{GI}_{50}$ values of seven derivatives in the test set were predicted (Table S4) and the residuals between the experimental data and the predicted values ranged from 2.15 to 13.19 . As a result, this model obtained here was concluded to be reliable. The contribution maps of the HQSAR model were generated as different colors, and their detailed explanation is described in the caption of Figure S6.

A natural polyphenol contained in green tea epigallocatechin-3-gallate acts as an apoptosis inducer by the DNA damage and a proliferation inhibitor by the reduction of
DNA synthesis in human SKOV-3 ovarian cancer cell lines [16]. The mechanism of a tetrahydroxy chalcone and butein inducing apoptosis in human ES-2 ovarian cancer cells has been reported [17]. Polyphenols known as components of black tea, theaflavin-3,3'-digallate, theaflavin-3'-gallate, theaflavin-3-gallate, and theaflavin inhibit angiogenesis in ovarian cancer cells, which were tested by enzyme-linked immunosorbent assay of vascular endothelial growth factor [18]. A polyphenol isolated from Combretum molle and punicalagin shows cytotoxic effects against human A2780 ovarian cancer cells via the inhibition of $\beta$-catenin signaling [19]. The study on quantitative relationships between polyphenols and the cisplatin-resistant ovarian cancer cells has been reported [6]. However, this study was performed on polyphenols containing cinnamaldehyde scaffold which are different from the current polyphenols with naphthalene skeleton. In addition, the current research adopted HQSAR method unlike the previous study where comparative molecular similarity index analysis method was used.

Based on the results obtained from the CoMFA model and the HQSAR model, pharmacophores to show better inhibitory effects against the growth of cisplatin-resistant A2780/Cis ovarian cancer cells can be derived as shown in Fig. 2. A direction of naphthalene attachment at C-3 of pyrazole is important. Naphthalene-2-yl was favored, while naphthalene-1-yl was not. A bulky substituent group at $N-1$ position is favored. The electron donating group such as sulfur of carbothioamide group and withdrawing groups at specific positions would increase the inhibitory effect against the cancer cell growth. The design and synthesis of novel polyphenols satisfying pharmacophores are remained for future study.

Acknowledgments This study was supported by the Basic Science Research Program through the National Research Foundation of Korea (NRF) funded by the Ministry of Science, ICT and Future Planning (Grant No. NRF-2016R1D1A1A09919045), Republic of 
Korea. This paper was supported by the KU Research Professor Program of Konkuk University.

\section{References}

1. Sudo T (2012) Molecular-targeted therapies for ovarian cancer: prospects for the future. Int J Clin Oncol 17:424-429

2. McGuire WP, Hoskins WJ, Brady MF, Kucera PR, Partridge EE, Look KY, Clarke-Pearson DL, Davidson M (1996) Cyclophosphamide and cisplatin compared with paclitaxel and cisplatin in patients with stage III and stage IV ovarian cancer. N Engl J Med 334:1-6

3. Galluzzi L, Vitale I, Michels J, Brenner C, Szabadkai G, HarelBellan A, Castedo M, Kroemer G (2014) Systems biology of cisplatin resistance: past, present and future. Cell Death Dis 5:e1257

4. Koberle B, Tomicic MT, Usanova S, Kaina B (2010) Cisplatin resistance: preclinical findings and clinical implications. Biochim Biophys Acta 1806:172-182

5. Usanova S, Piée-Staffa A, Sied U, Thomale J, Schneider A, Kaina B, Köberle B (2010) Cisplatin sensitivity of testis tumour cells is due to deficiency in interstrand-crosslink repair and low ERCC1-XPF expression. Mol Cancer 9:248

6. Shin SY, Jung H, Ahn S, Hwang D, Yoon H, Hyun J, Yong Y, Cho HJ, Koh D, Lee YH, Lim Y (2014) Polyphenols bearing cinnamaldehyde scaffold showing cell growth inhibitory effects on the cisplatin-resistant A2780/Cis ovarian cancer cells. Bioorg Med Chem 22:1809-1820

7. Yoon H, Ahn S, Park M, Kim DW, Kim SH, Koh D, Lim Y (2013) $1 \mathrm{H}$ and 13C NMR spectral assignments of chalcones bearing pyrazoline-carbothioamide groups. Magn Reson Chem 51:500-508

8. Hwang D, Hyun J, Jo G, Koh D, Lim Y (2011) Synthesis and complete assignment of NMR data of 20 chalcones. Magn Reson Chem 49:41-45
9. Hwang D, Jo G, Hyun J, Lee SD, Koh D, Lim Y (2012) Synthesis of methoxybenzoflavones and assignments of their NMR data. Magn Reson Chem 50:62-67

10. Shin SY, Yoon H, Ahn S, Kim DW, Bae DH, Koh D, Lee YH, Lim Y (2013) Structural properties of polyphenols causing cell cycle arrest at G1 phase in HCT116 human colorectal cancer cell lines. Int J Mol Sci 14:16970-16985

11. Jung Y, Shin SY, Yong Y, Jung H, Ahn S, Lee YH, Lim Y (2015) Plant-derived flavones as inhibitors of aurora B kinase and their quantitative structure-activity relationships. Chem Biol Drug Des 85:574-585

12. Jung KY, Park J, Han YS, Lee YH, Shin SY, Lim Y (2017) Synthesis and biological evaluation of hesperetin derivatives as agents inducing apoptosis. Bioorg Med Chem 25:397-407

13. Lee Y, Kim BS, Ahn S, Koh D, Lee YH, Shin SY, Lim Y (2016) Anticancer and structure-activity relationship evaluation of 3-(naphthalen-2-yl)-N,5-diphenyl-pyrazoline-1-carbothioamide analogs of chalcone. Bioorg Chem 68:166-176

14. Cho M, Yoon H, Park M, Kim YH, Lim Y (2014) Flavonoids promoting HaCaT migration: I. Hologram quantitative structureactivity relationships. Phytomedicine 21:560-569

15. Kim BS, Shin SY, Ahn S, Koh D, Lee YH, Lim Y (2017) Biological evaluation of 2-pyrazolinyl-1-carbothioamide derivatives against HCT116 human colorectal cancer cell lines and elucidation on QSAR and molecular binding modes. Bioorg Med Chem 25:5423-5432

16. Rao SD, Pagidas K (2010) Epigallocatechin-3-gallate, a natural polyphenol, inhibits cell proliferation and induces apoptosis in human ovarian cancer cells. Anticancer Res 30:2519-2523

17. Yang PY, Hu DN, Lin IC, Liu FS (2015) Butein shows cytotoxic effects and induces apoptosis in human ovarian cancer cells. Am J Chin Med 43:769-782

18. Gao Y, Rankin GO, Tu Y, Chen YC (2016) Inhibitory effects of the four main theaflavin derivatives found in black tea on ovarian cancer cells. Anticancer Res 36(2):643-651

19. Tang JM, Min J, Li BS, Hong SS, Liu C, Hu M, Li Y, Yang J, Hong L (2016) Therapeutic effects of punicalagin against ovarian carcinoma cells in association with $\beta$-catenin signaling inhibition. Int J Gynecol Cancer 26:1557-1563 